\title{
Michel Van der Yeught, L'anglais de la bourse et de la finance : description et recherche
}

Paris : Éditions Ophrys, 2012

\section{Martine Azuelos}

\section{CpenEdition}

\section{Journals}

Édition électronique

URL : http://journals.openedition.org/asp/3276

DOI : $10.4000 /$ asp.3276

ISSN : 2108-6354

\section{Éditeur}

Groupe d'étude et de recherche en anglais de spécialité

Édition imprimée

Date de publication : 1 mars 2013

Pagination : 169-172

ISSN : 1246-8185

\section{Référence électronique}

Martine Azuelos, «

Michel Van der Yeught, L'anglais de la bourse et de la finance : description et recherche », ASp [En ligne],

63 | 2013, mis en ligne le 01 mars 2013, consulté le 02 novembre 2020. URL : http://

journals.openedition.org/asp/3276 ; DOI : https://doi.org/10.4000/asp.3276

Ce document a été généré automatiquement le 2 novembre 2020.

Tous droits réservés 
Michel Van der Yeught, L'anglais de la bourse et de la finance : description et recherche

Paris : Éditions Ophrys, 2012

Martine Azuelos

\section{RÉFÉRENCE}

Van der Yeught, Michel. 2012. L'anglais de la bourse et de la finance : description et recherche. Paris : Éditions Ophrys, collection Langues de spécialité. 320 pages. ISBN 9-782-7080-1330-8. 
1 Cet ouvrage est le premier de la collection «Langues de spécialité » dirigée par Michel Van der Yeught aux éditions Ophrys en partenariat avec le GERAS et le LERMA (EA 853) de l'Université d'AixMarseille. Son intérêt réside donc tout autant dans la présentation de la langue de spécialité sur laquelle portent sa deuxième et sa troisième partie (l'anglais de la bourse et de la finance) que dans la vingtaine de pages introductives consacrées à la présentation de la collection et du cadre théorique (première partie) qui en définit la philosophie.

2 L'introduction, qui souligne l'originalité de la collection «Langues de spécialité » dans le paysage éditorial français, permet d'emblée de saisir les enjeux de son

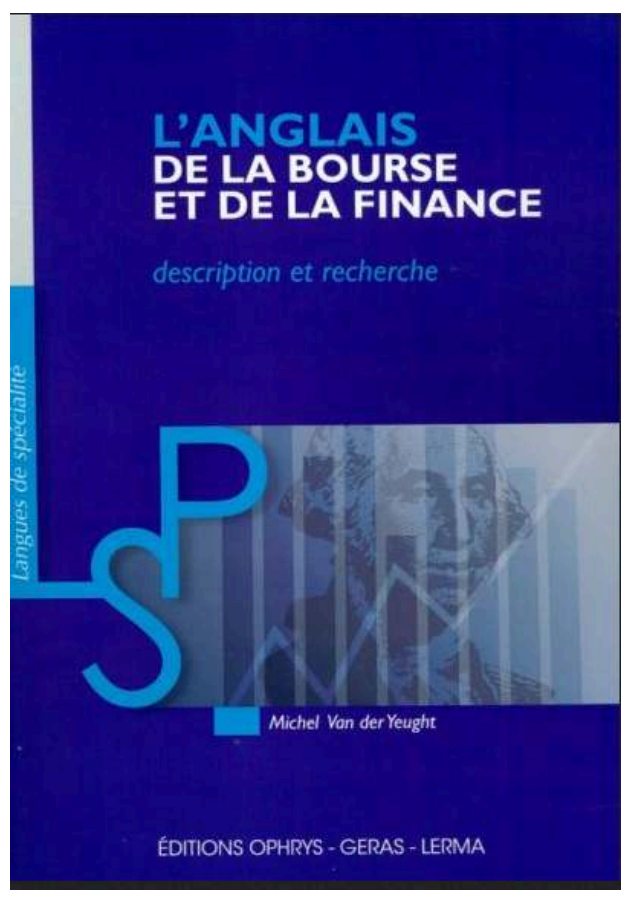
lancement. Il s'agit en effet de répondre à « une demande sans offre » dans le secteur Lansad. Rappelant l'ampleur, la croissance rapide et l'hétérogénéité de ce secteur jusqu'aux années 2000, M. Van der Yeught indique aussi que la mise en place du LMD a imposé une rationalisation des programmes et l'incorporation en leur sein d'une part croissante de contenus spécialisés pour que la formation linguistique des étudiants soit cohérente avec l'allongement de leur formation disciplinaire et sa professionnalisation progressive. Or si ces évolutions créent une forte demande de linguistes spécialisés, force est de constater que l'offre reste, dans ce domaine, largement inadaptée: la plupart des formateurs de Lansad sont des généralistes, titulaires du CAPES ou de l'agrégation, qui se forment sur le tas en fonction des composantes dans lesquelles ils sont appelés à enseigner. C'est à ces formateurs, ainsi qu'à ceux qui souhaitent s'engager dans une recherche dans le domaine des langues de spécialité (LSP) que sont destinés les titres de la collection. Pour chaque variété de LSP étudiée, deux volumes seront mis à leur disposition: un volume "description et recherche » destiné aux enseignants-chercheurs et un manuel destiné à leurs étudiants. Les volumes « description et recherche » seront tous structurés de façon identique. Le texte de présentation de la collection et la première partie qui en définit le cadre théorique, que l'on trouve ici, seront communs à tous les titres, ce qui conférera son unité à la collection. Une seconde partie sera, ensuite, consacrée à la description de la langue concernée. Elle traitera de ses origines et de ses évolutions, mais aussi des différentes approches (linguistique, discursive et culturelle) par lesquelles se structure son étude, chacune de ces approches étant illustrée par des articles de recherche. Une troisième partie, enfin, regroupera les ressources et les références permettant un approfondissement du sujet.

3 Quel est donc le cadre théorique qui donne corps à cette collection? L'enjeu est d'importance car, comme le souligne l'auteur, les LSP « se présentent comme des objets complexes malaisés à appréhender » et " un consensus sur leur nature reste encore à construire » (p. 9). Pour faire progresser le débat, la collection envisage les LSP comme 
« des objets d'étude en soi à l'intersection entre une langue et un domaine de spécialité donné » (p.10). Plus précisément, « [u]ne LSP ne réside ni dans la langue générale ni dans la spécialité, mais dans les relations permanentes qui se tissent entre ces deux ordres de réalité ». En conséquence, «[1]e spécialiste de LSP n'est ni un expert de la langue générale ni un expert de la spécialité ; c'est un spécialiste de ces relations où la spécialité s'exprime dans la langue » (p.12). Après avoir pris connaissance des outils conceptuels et des définitions qui serviront de fondements théoriques à la description des LSP, le lecteur est initié à la méthode consistant à aborder cette description par le biais de la langue, des discours et de la civilisation. C'est en effet cette combinaison qui va permettre de dessiner le profil global de chaque LSP. Angliciste lui-même, M. Van der Yeught ancre cette démarche théorique dans les travaux pionniers de spécialistes de l'anglais de spécialité comme Michel Petit ou Catherine Resche. Il ajoute que, dans la plupart des cas, le spécialiste de LSP ne pourra se passer de l'apport des études de civilisation. La méthode proposée permet de rendre compte de discours spécialisés porteurs d'une dimension civilisationnelle en même temps qu'elle contribue à l'émergence d'un nouveau domaine transdisciplinaire se situant à l'intersection des langues de spécialité et de la civilisation. On est loin, on le voit, des pratiques qui se sont développées dans le monde anglo-saxon dans le cadre de l'enseignement de l' English for Special Purposes (ESP). Pour marquer la spécificité et l'apport épistémologique de la démarche qu'entend promouvoir la collection «Langues de spécialité», la première partie conclut sur un rappel des pratiques de l'ESP dont les mérites, mais aussi les limites, sont soulignés.

4 La deuxième et la troisième partie de l'ouvrage, consacrées à l'anglais de la bourse et de la finance, constituent une première illustration de la démarche proposée par la collection et en confirment l'intérêt. Ces parties sont de longueur très inégale, la troisième («Ressources et références ») comptant une vingtaine de pages, la deuxième plus de 250. Véritable cœur de l'étude, la seconde partie revient en premier lieu sur les conditions d'émergence de l'anglais de la bourse. Si cette variété spécialisée de l'anglais a en effet vu le jour, en tant que langue spécialisée, dans l'Angleterre du début du XVIII ${ }^{\mathrm{e}}$ siècle, ce n'est que dans les années 1980 que les chercheurs anglicistes français ont commencé à s'y intéresser, en réponse au développement de l'activité de la City et de Wall Street. C'est alors qu'elle s'est constituée en une LSP qui reflète les multiples complexités du domaine spécialisé qui s'y exprime. Revenant sur plus de trois siècles d'histoire anglo-américaine (de la construction du Royal Exchange de Londres [1567] au Gilded Age), le chapitre 2 montre comment un processus de dictionnarisation a progressivement extrait cette langue du contexte discursif pour la rendre accessible au non-spécialiste. Aujourd'hui encore, les approches linguistiques de l'anglais de la bourse et de la finance restent essentiellement lexicale et terminologique, comme le montrent les documents de recherche illustrant le chapitre 3. Plus récentes sont les approches discursives, qui s'avèrent particulièrement fécondes en ce début de $\mathrm{XXI}^{\mathrm{e}}$ siècle en raison de l'intensité de la production de discours et des échanges communicationnels par les communautés spécialisées dont relève l'anglais de la bourse et de la finance. Si ces approches discursives ont en commun d'aborder cette LSP en faisant référence au contexte et à la civilisation dans laquelle elle s'inscrit, la richesse du champ, qui tient largement au caractère inépuisable et constamment renouvelé des supports, favorise la diversité des perspectives. Diversité dont le chapitre 4, de loin le plus long de l'ouvrage (116 pages) s'attache à rendre compte. Distinguant les approches selon qu'elles privilégient l'analyse des métaphores, l'analyse rhétorique et stylistique, 
l'étude par genres (hors FASP et FASP), ou enfin un genre propre au domaine, le Wall Street classic, la typologie proposée et les textes de recherche choisis pour l'illustrer permettent de saisir la richesse, la finesse et la qualité des éclairages apportés par ces analyses. Le chapitre suivant laisse en revanche le lecteur un peu sur sa faim. Si la légitimité du troisième niveau d'approche des LSP, l'approche "culturelle ", à laquelle il est consacré apparaît tout à fait légitime, sa mise en œuvre ne semble pas pleinement aboutie. Partant du postulat que les LSP sont le moyen d'expression de certaines visions collectives du monde partagées par les parties prenantes au domaine spécialisé, l'auteur avance que l'étude de l'anglais de la bourse et de la finance gagne à être alimentée par la connaissance de l'histoire et l'étude des cultures en tant qu'elles reposent sur des valeurs partagées par un même groupe. L'illustration qu'en fournissent les documents 10,11 et 12 cités dans ce chapitre n'en épuise pas pour autant ce vaste sujet, qui a d'ailleurs déjà été abordé par un autre angle, celui de l'analyse des discours, dans le chapitre précédent. L'apport de l'étude de l'anglais de la bourse et de la finance à l'étude de la civilisation britannique et américaine et à son enseignement dans un contexte universitaire est un point important qui aurait pu, lui aussi, être plus développé.

5 En terminant sa deuxième partie (chapitre 6) sur un bilan qui ne saurait apporter de conclusion définitive à la description de la LSP à laquelle est consacrée l'ouvrage, M. Van der Yeught va au-devant de ces quelques réserves en soulignant tout à la fois l'apport de son étude et la nécessité qu'elle soit prolongée et enrichie par d'autres. Si l'ouvrage a en effet permis de dénombrer dix caractéristiques essentielles lui permettant de dresser le profil de l'anglais de la bourse et de la finance (p. 273-274), « [à] ce stade, la langue a été étudiée par un nombre insuffisant de chercheurs pour que la description ébauchée ici recueille un consensus scientifiquement acceptable. Les avancées réalisées doivent être poursuivies et reconsidérées, de nombreuses autres approches doivent être engagées, de nombreux outils conceptuels doivent être forgés par une communauté d'auteurs plus nombreuse et diversifiée ». Comment ne pas souscrire aux perspectives d'avenir pour la recherche sur l'anglais de la bourse et de la finance que dessine cette conclusion? Loin que la crise financière déclenchée en 2008 ait mis un terme aux «Trente Glorieuses de Wall Street» et que la contestation incarnée par le mouvement Occupy ait débouché sur une remise en cause radicale de la financiarisation de l'économie, il semble bien que la finance doive rester quelque temps encore au cœur de l'activité en Grande-Bretagne, aux États-Unis, mais aussi dans une économie mondialisée dont l'anglais de la bourse et de la finance est devenu, de fait, la lingua franca.

6 On ne doute pas que cet ouvrage fera date. Chercheurs ou enseignants spécialisés dans l'anglais de la bourse et de la finance, et plus largement en anglais économique, y trouveront une méthode d'approche innovante et stimulante appliquée à un domaine encore insuffisamment exploré par la recherche. Les articles rassemblés pour illustrer les différentes approches sont de nature à alimenter une réflexion scientifique qui pourra être prolongée dans le cadre des sociétés savantes, groupes de recherche et revues référencées dans la troisième partie, mais aussi, espérons-le, dans le cadre de recherches doctorales. L'abondante bibliographie et la filmographie figurant à la fin du volume constituent une mine pour quiconque souhaite s'engager dans une recherche dans ce domaine ou dans un domaine connexe. Cependant, la limpidité du style, le caractère méthodique de la démarche et la clarté de l'argumentation rendent l'ouvrage accessible au non-spécialiste. Il est donc permis de penser que le cadre théorique 
original élaboré par Michel Van der Yeught et le lancement de la collection «Langues de spécialité » ne devraient pas manquer de susciter l'intérêt d'un large public, tant en France qu'à l'étranger.

\section{AUTEURS}

\section{MARTINE AZUELOS}

Université Sorbonne Nouvelle Paris 3 